\title{
APPLICATION OF 3D MODEL OF CULTURAL RELICS IN VIRTUAL RESTORATION
}

\author{
Sizhong Zhao ${ }^{1}$, Miaole Hou ${ }^{1,2,3} *$, Yungang Hu ${ }^{1,2,3 *}$, Qi Zhao ${ }^{1}$ \\ ${ }^{1}$ School of Geomatics and Urban Spatial Informatics, Beijing University of Civil Engineering and Architecture, No.15Yongyuan \\ Road, Daxing District, Beijing, 102616-(zhaosizhong, houmiaole, hyg, zhaoqi)@bucea.edu.cn \\ ${ }^{2}$ Beijing Key Laboratory For Architectural Heritage Fine Reconstruction \& Health Monitoring, No.15Yongyuan Road, Daxing \\ District, Beijing, 102616 \\ ${ }^{3}$ Engineering Research Center of Representative Building and Architectural Heritage Database, Ministry of Education, \\ No.15Yongyuan Road, Daxing District, Beijing, 102616
}

Commission III, WG III/5

KEY WORDS: 3D Model, Cultural Relics Protection, Terracotta Warriors Debris, Significant Features, Virtual Restoration

\begin{abstract}
:
In the traditional cultural relics splicing process, in order to identify the correct spatial location of the cultural relics debris, experts need to manually splice the existing debris. The repeated contact between debris can easily cause secondary damage to the cultural relics. In this paper, the application process of 3D model of cultural relic in virtual restoration is put forward, and the relevant processes and ideas are verified with the example of Terracotta Warriors data. Through the combination of traditional cultural relics restoration methods and computer virtual reality technology, virtual restoration of high-precision 3D models of cultural relics can provide a scientific reference for virtual restoration, avoiding the secondary damage to the cultural relics caused by improper restoration. The efficiency and safety of the preservation and restoration of cultural relics have been improved.
\end{abstract}

\section{INTRODUCTION}

Cultural relics are relics and remains of historical, artistic, and scientific value created by the human wisdom and labor in the development and evolution of the historical society. They are numerous in number, rich in variety, and diverse in texture. They represent the cultural and wisdom of a nation, is the symbol of the development level and trend of different historical periods of economic and political, science and technology and culture, and provide a reliable basis and very precious material for modern people to understand and study history. Due to human activities, environmental changes, natural disasters, protection and other reasons, a large number of cultural relics are rapidly disappearing and damaged in an irreversible manner. Therefore, applying modern science and technology to effectively inherit and protect precious cultural relics have made human civilization better, has become a common problem that human society must face. (Fabio, 2010; Anna, 2012; Andrey, 2014; Shan, 2017)

In the archaeological excavation, every site will unearthed a large number of fragmented and broken cultural relics. For workers to complete the restoration of cultural relics, they often need to do a series of cumbersome and complicated manual repair procedures such as recording, numbering, storing, measuring, analyzing, comparing, drawing, and assembling. Because the human eye and brain often show poor recognition and memory when dealing with large amounts of data, when there are many fragments or their shapes are complex and varied, even professional cultural relics workers with rich cultural relics restoration experience, it is difficult to accurately determine the adjacency relationship between fragments. At the same time, a series of operations in manual repair can not avoid frequent contact and friction with cultural relics. It is easy to cause secondary damage to cultural relics while there is a certain damage in the artifacts. Traditional technical means can no longer provide effective scientific support for the preservation and restoration of cultural relics. $(\mathrm{Li}, 2017)$

Therefore, how to use new technologies to provide accurate, detailed, scientific data and support for cultural relics protection has become a major demand for cultural relics protection in recent years. With the development of technologies such as digital photogrammetry, laser scanning, 3D model and visualization, digital preservation of cultural relics has become an important scientific research direction all over the world. Scholars at home and abroad have obtained certain research achievements and related accumulation in the preservation of cultural relics, such as 3D information preservation, highprecision 3D modeling, and so on, making it possible to digitally protect relevant cultural relics based on high-precision 3D models. (Koller, 2006; Lucia, 2013; Andrey, 2014; Cemoli, 2017; Huang, 2007; Zhang, 2007; Yang, 2012; Hou, 2015) Combined with traditional cultural relics restoration method and virtual reality technology can provide a scientific reference for restoration work, solve the difficult problem of cultural relic debris, to avoid improper repair cause secondary damage to cultural relics. (Chalmoviansky, 2003; Noorudin, 2003; Ju, 2004; Yu, 2005; Fabio, 2010; Anestis, 2011; Anna, 2012; Lerones, 2014; Pan, 2003; Zhou, 2014; Liu, 2017; Zhang, 2017) The virtual restoration of cultural relics provides an effective auxiliary means for the research, restoration and Archaeology of cultural relics, which is an important embodiment of the role of information technology in understanding ancient cultures.

\footnotetext{
* Corresponding author. E-mail addresses: houmiaole@ bucea.edu.cn (Miaole Hou), hyg@ bucea.edu.cn (Yungang Hu).
} 


\section{METHOD AND PROCESS}

\subsection{D Model Application of Cultural Relics}

The digital information retention of 3D information is a method to study the rapid acquisition of the spatial coordinate of the surface and to obtain the 3D digital model of the object. In the 1990s, 3D laser scanning technology provided a new technological means for the acquisition of spatial 3D information, it overcomes the limitations of traditional measurement techniques and uses non-contact, active measurement methods to acquire a large number of highprecision 3D coordinates. It can perform surface scanning on any visible object and is not limited by day and night. It can quickly convert the real-world spatial information into $3 \mathrm{D}$ data that can be easily processed by computers.

With the development of 3D scanning technology, the accuracy and speed of scanning data have been greatly improved, and the instrument and equipment are more and more portable. Therefore, 3D scanning technology has been extended from traditional industry to the field of cultural relics protection. Using this technology, the cultural relics are scanned to obtain massive point cloud data on the surface of the cultural relics. After the pre-processing of the point cloud data, such as noise reduction, etc., the cultural relics can be generated in 3D lines, surfaces, and body shapes. Based on the detailed modeling of point cloud data, comprehensive application of cultural relics protection can be further carried out, such as analysis, simulation, monitoring, simulation, virtual reality and so on. (Figure 1)

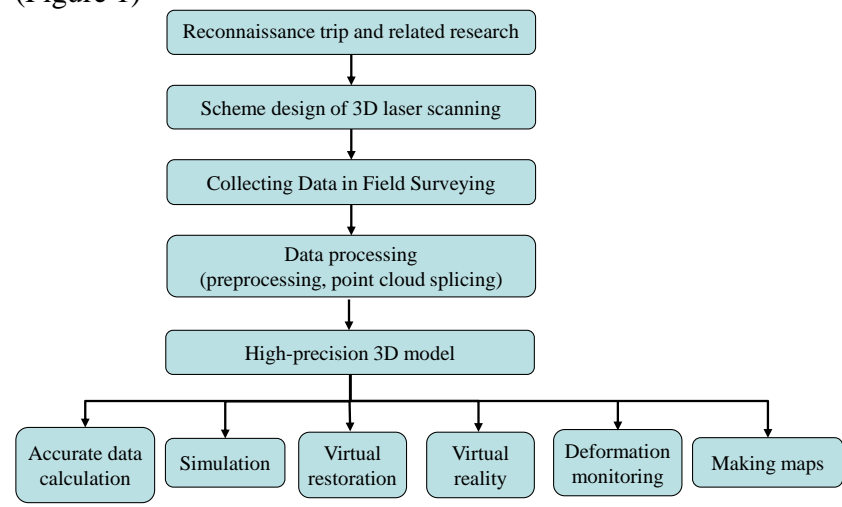

Figure 1 General working process of three dimensional digitalization of cultural relics

The most widely used 3D model of cultural relics in the protection of cultural relics is to build up 3D digital models of all kinds of cultural relics by advanced laser scanning technology, digitally record and preserve the cultural relics. Since the data obtained from the 3D scanning is the most original accurate size data of cultural relics, the length and area of any position can be measured in the model, and also can understand the geometric characteristics of the cultural relics and the damage of the disease in an all-round way. And the non-contact of the scanning mode makes it impossible to cause secondary damage to the original when acquiring data. Therefore, 3D model built by 3D laser scanning data can be used for virtual restoration of cultural relics through 3D processing software, and according to the effect of virtual restoration, it will provide reference and guidance for cultural experts to formulate a practical restoration plan. Taking Mausoleum of the First Qin Emperor Terracotta Warriors restoration project as an example.
The introduction of digitalized technology in the protection of cultural relics is a technological revolution in the field of cultural relics protection, which will change the traditional technology and means of cultural relics protection, and even affect the way of thinking and workflow of archaeologists. The emergence of 3D digital protection technology for cultural relics has opened up a new direction for the protection of cultural relics. The application of the technology in surveying and mapping in the cultural relics can not only obtain the surface data quickly and accurately, but also does not need to contact the body of the cultural relics in the scanning process, and will not cause secondary damage to cultural relics. This technology has greatly changed the method of surveying and mapping cultural relics. It provides a complete spatial information base for the establishment of a comprehensive system for the protection of cultural relics.

\subsection{Process of Virtual Restoration}

In the traditional cultural relics splicing process, in order to identify the correct spatial location of cultural relics, cultural relics experts often attempt to attempt to splice multiple pieces of existing cultural relics. A great deal of contact can easily cause secondary damage to the cultural relics. When the Terracotta Warriors pottery were unearthed, the surface is painted texture, so excessive contact between the debris will cause painted shedding, peeling and other phenomena. The virtual restoration based on the 3D model of Terracotta Warriors can avoid secondary irreversible damage caused by repeated comparison and splicing debris during the actual repair process. (Figure 2)

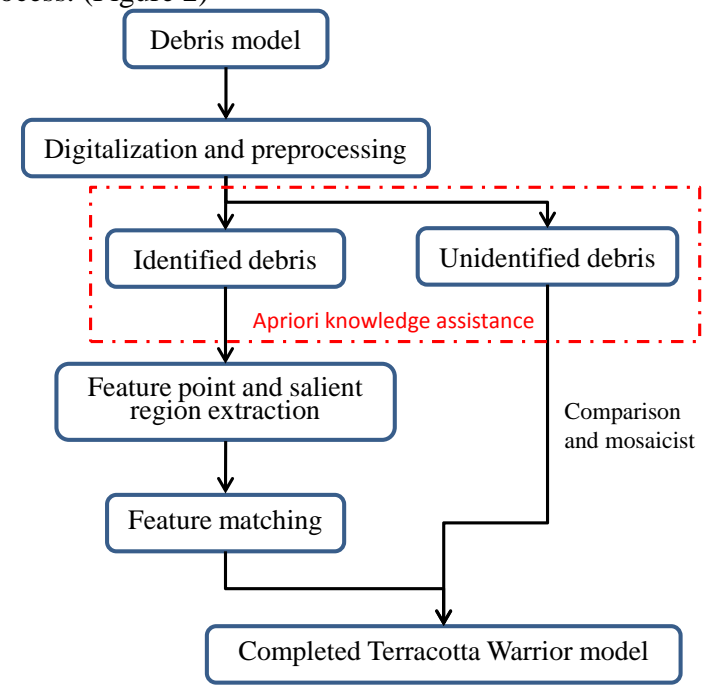

Figure 2. Virtual restoration flow of Terracotta Warriors

The damaged surface of the Terracotta Warriors debris are characterized by high noise, non-smooth and so on. In space geometry, the curvature with translational rotation invariance can describe the essential characteristics of the surface. The multi-scale feature quantity defined by the model's vertex local neighborhood integral not only weakens the influence of noise when describing the curvature, but also is more robust than the micro component. The extraction method is presented significant geometric feature discretization artifacts adjacency matrix constructed by integral coordinates based on vertex expansion algorithm, cluster analysis was performed on all vertices integral invariant values, using integral invariants of $3 \mathrm{D}$ model feature extraction was Terracotta Army. Therefore, a 
method for extracting the significant geometric features of the discrete coordinate of the cultural relics based on the adjacency matrix is proposed. The vertex expansion search algorithm is used to cluster analysis the values of all vertex integral invariants of the model, using the integral invariant to extract the significant features of the Terracotta Warriors 3D model The extension search is performed through the topological relations among adjacency matrix vertexes. If a vertex searches for similar points in its neighborhood, it continues to expand outwards, and gradually expands from feature points to feature lines and feature planes. On the other hand, if the same point is not searched in a certain neighborhood, the point can be identified as a significant feature point. Finally, the geometric features such as feature points, feature lines, and feature surfaces are generated.

The characteristics of most of the existing measure of model similarity matching algorithm is directly based on the feature points, that is, calculating some local geometric features of vertices and extracting feature vertices as feature points, and then looking for the similarity points among the model surface in a given model library, and finally similarity measures are made by measuring the matching degree of similarity points. However, the fracture surface of Terracotta Warriors debris model is relatively complex, and the number of vertex pairs with similar neighborhood information is huge. For a certain vertex, there is only one true matching point on the surface of the other model, and the rest are false matching points. The search space domain is very large when there is an optimal matching, and the speed and accuracy of the matching are greatly affected. Combining the extracted geometric features (points, lines, planes), it is clear that this contains more information than the neighborhood information of a single feature point. In order to achieve the similarity calculation of 3D models of cultural objects based on significant geometric features, based on independent component analysis and multilayer local ICP algorithm, the model similarity of several feature levels was obtained, and weighted by RMS and multiple factors. The similarity calculation results are corrected to obtain the final result of similarity. (Figure 3 ) it is more reliable to carry out model similarity with significant geometric features, and can achieve partial matching.

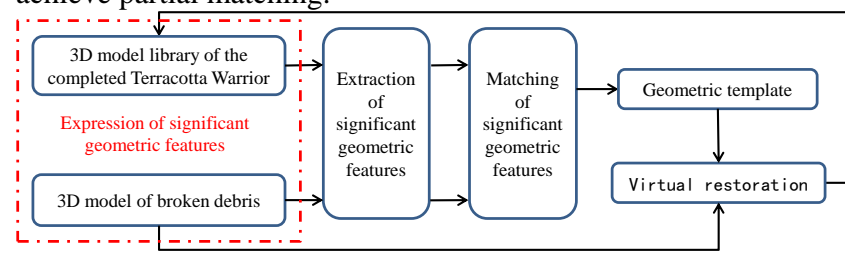

Figure 3. Similarity calculation of 3D model of debris based on significant geometric features

\section{VIRTUAL RESTORATION BASED ON 3D MODEL}

\subsection{Experimental Data and Processing}

The Terracotta Warriors, known as the "eighth wonder of the world" were discovered in the east of the Qin Shihuang Mausoleum in the foothills of Mount Li in Xi'an in 1974. During the excavation work of the Qin Shi Huang Mausoleum, most have been broken into different sizes, different shapes of pottery pieces, rarely complete pottery unearthed. (Figure 4)

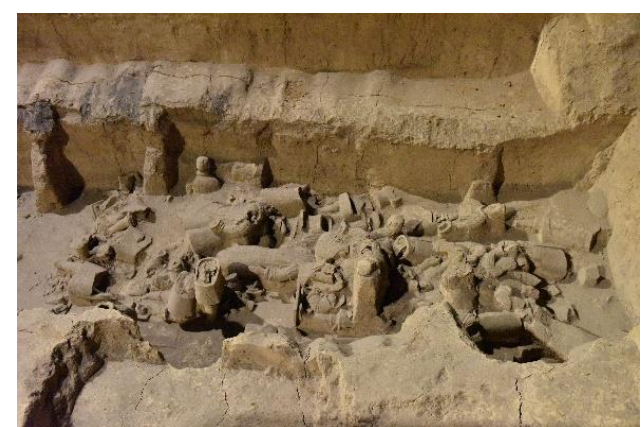

Figure 4. The excavation site of Terracotta Warriors

Therefore, how to manage and protect a large number of unearthed Terracotta pottery pieces and provide important data and theoretical support for the research and actual restoration work of the Terracotta Warriors is an important issue that archeologists urgently need to solve at this stage.

In this project, Hexagon's hand-held articulated arm 3D laser scanner was used to perform for the G11-33 figurines cultural relics debris unearthed in Pit No.1 of the Terracotta Warriors of Qin Shihuang Mausoleum, and acquire high precision real information of a large number of terracotta pottery. (Figure 5)

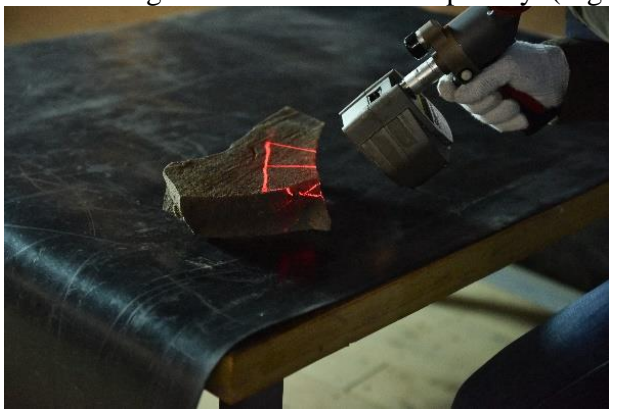

Figure 5. 3D laser scanning of the Terracotta Warriors debris

Due to the precision error and human disturbance of the digital equipment, the acquired 3D data inevitably contains some noise, the original features of the model are blurred, lost, or even distorted, and the presence of noise is not conducive to subsequent operations such as virtual restoration of the model, the first to point clouds denoising. High precision 3D laser scanner to scan the data acquisition model will produce redundant data, and too large will reduce the efficiency of subsequent model processing and increase the complexity of virtual repair operations. Therefore, it is necessary to maintain a model of the original shape and the main features, the model is simplified. Figure 6 is the use of the point cloud data and digital images have been obtained on the Terracotta Film (No. G11-33 head figurines) carried out three-dimensional model of the true color of the head to achieve digital preservation of the unearthed cultural relics.

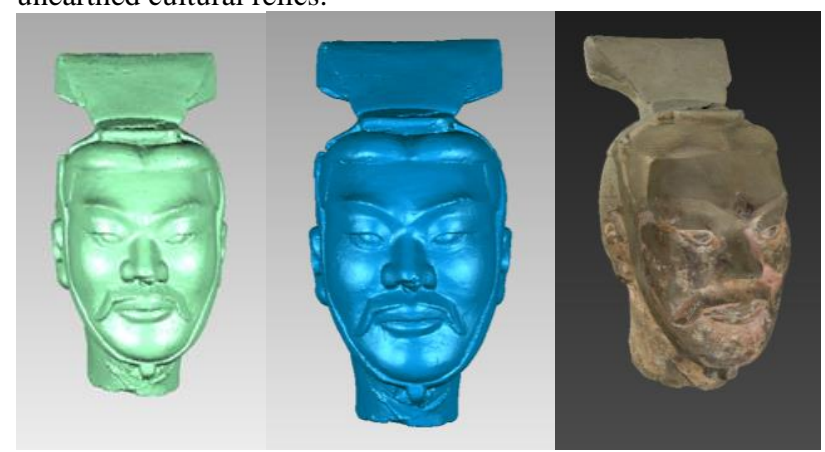


(a)

(b)

(c)

Figure 6. No. G11-33 the digital preservation of the head of the Terracotta Warriors

(a) Point cloud model; (b) Triangulation model; (c) True color model

After constructing a fine 3D model, the 3D model of the Terracotta Warriors is extracted using the integral invariant to extract significant features and expand the search through the topological relationship among the adjacency matrix vertices, generating characteristic features such as feature points, feature lines, and feature planes. Figure 7 shows the results of the significant feature extraction of the G11-33 Terracotta Warriors shoes debris model, which is marked in red and blue.

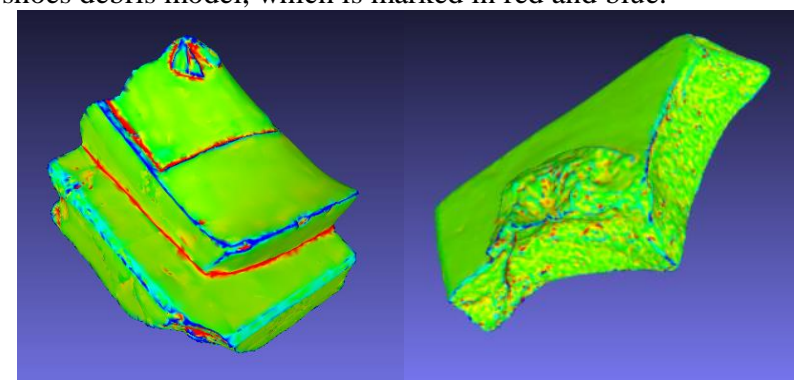

Figure 7. Schematic diagram of significant geometric features

\subsection{Construction of Debris Splicing Software}

In the traditional restoration and splicing process of the Terracotta Warriors debris, the space location of the debris is determined by manually and repeatedly trying to pair each other. In order to improve the stitching speed and accuracy, the repair staff will classify the excavated debris in advance before stitching. Even so, it takes at least 3 5 months for a full spell of Terracotta Warriors. It not only takes time and effort, but also the debris is contacted several times during the process of determining the mutual relations, and the surface of the painted texture will also be shed and peeled. Based on this proposal, a 3D model splicing software for the Terracotta Warriors debris is constructed, unearthed pottery fine 3D model of the current access, due to the difference in the size of the debris, the size of the 3D model data will also be different. So most of the Splicing repair software is facing with how to simplify the model data but retains its significant feature information to ensure that subsequent matching can be achieved.

Therefore, based on the 3D model of high-precision orthophoto and sub-millimeter fine-grained artifacts excavated from the Terracotta Warriors, the process of virtual stitching for multilayered cultural relics in rough fine stitching was studied, and a practical and efficient 3D stitching software system was developed to realize cultural relics. The splicing of debris and visualization of the virtual restoration of cultural relics ensure the scientificity and rigor of the restoration of cultural relics.

The process of virtual splicing of multi-level cultural debris is to first import the refined 3D model of sherds into the selfdeveloped 3D splicing recovery software, and then introduce the domain expert's prior knowledge to assist the computer to identify and number the unearthed sherd classification (for example, Pieces with armor straps and nails are armor pieces; relatively smooth pieces may be parts of the shirt; more prominent features such as head, arms, legs, shoes, etc.).
After classifying and identifying the debris, we use the mosaic algorithm based on the extraction of significant geometric features to complete the "roughly splicing" in the self-developed software, forming a general outline area of Terracotta Warriors. After the combination of human and machine, by manually picking the unclassified marks and the debris that were not identified in the "rough stitching" and then splicing in the contour area already formed, they try each other, that is, "fine stitching", and finally get accurate. After the completion of the whole, the digital 3D model of the Terracotta Warriors, which determines the spatial relationship among all the debris, can be obtained. (Figure 8). The restoration workers can quickly and efficiently repair the terracotta warriors and horses based on the debris number in the actual splicing work, and at the same time reduce the secondary damage caused by repeated attempts to split the terracotta debris.

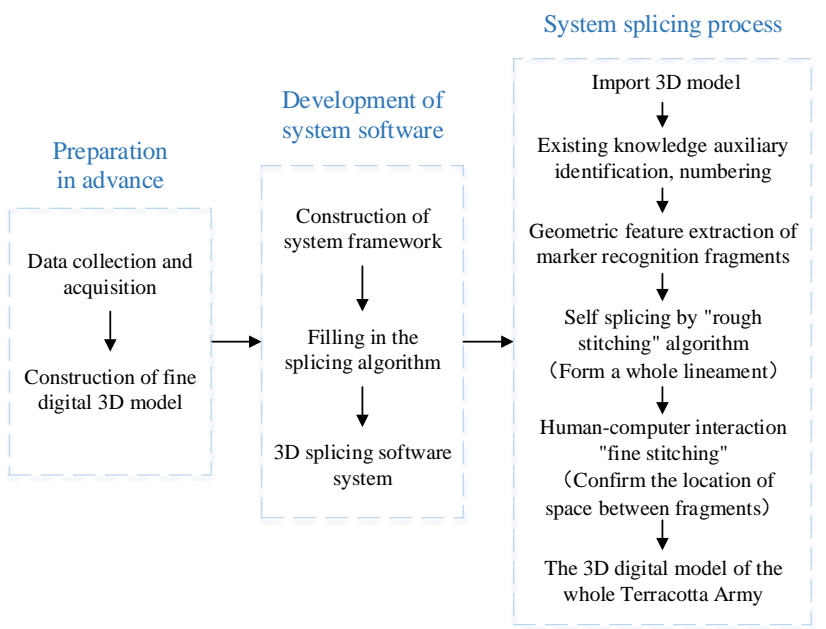

(a)

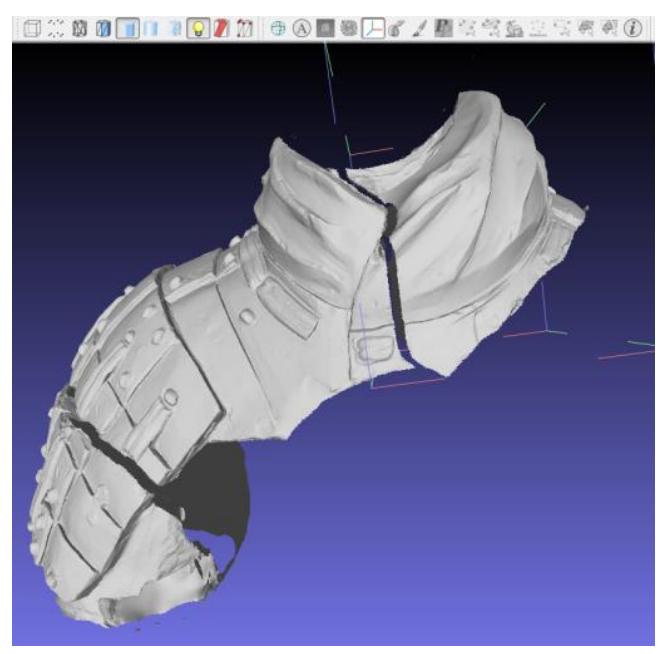

(b)

Figure 8. 3D model stitching software

(a) The development and workflow of the system; (b) Display of interface and splicing effect

The successful solution of this problem will open up new applications of pattern recognition and promote the development of digital image processing, pattern recognition, $\mathrm{AI}$ and other fields, and will also be applied to new fields in the future. 


\section{CONCLUSION}

In this paper, the application process of 3D model of cultural relic in virtual restoration is put forward, and the relevant processes and ideas are verified with the example of Terracotta Warriors data. There are two main tasks: 1. A method for extracting significant geometric features based on integral invariants is proposed and used to measure feature similarity for of the model; 2. and based on the high precision of Mausoleum of the First Qin Emperor Terracotta Army unearthed pottery orthophoto and fine 3D model. Research on the process of virtual stitching of multi-level cultural relic debris with rough stitching and fine stitching, and development of efficient 3D stitching software system. The virtual restoration technology combined with traditional cultural relics restoration method and virtual reality technology can provide a scientific reference for restoration work, solve the difficult problem of cultural relic debris, to avoid improper repair cause secondary damage to cultural relics, provide scientific and technological support for the restoration of cultural relics, improve the efficiency and safety of cultural relics protection and restoration, has strong theoretical and application value.

\section{REFERENCES}

Andrey, V., Mikhail, N., Ivanov, A., et al., 2014.Laser scanning and 3D modeling of the Shukhov hyperboloid tower in Moscow. Journal of Cultural Heritage, 16(4), pp. 551-559.

Anestis, K.,Christodoulos, C., 2011. 3D pottery shape matching using depth map images. Journal of Cultural Heritage, 12(2), pp.128-133.

Anna, M., Valentina, B., Cristiana, C.,2012.An integrated and automated segmentation approach to deteriorated regions recognition on 3D reality-based models of cultural heritage artifacts. Journal of Cultural Heritage, 13(4),pp.371-378.

Cemoli, L., D’Auria, S., Silla, F. D., Pucci, S., \& Strollo, R. M. (2017): Infographic modeling based on $3 d$ laser surveying for informed universal design in archaeological areas: the case of oppidum of the ancient city of tusculum. , IV-2/W2,pp. 259-264

Chalmoviansky, P., Jüttler, B.,2003.Filling holes in point clouds. Spring Berlin Heidelberg, 2768,pp.196-212.

Fabio, B., Stefano, B., Giovanna, S., et al.,2010. From 3D reconstruction to virtual reality: A complete methodology for digital archaeological exhibition Original. Journal of Cultural Heritage, 11(1),pp.42-49.

Hou M.L., Yang S., Hu Y.G., et al., 2015.A novel method for the virtual restoration of cultural relics based on a 3D fine model. Dyna, 90(3),pp.307-313.

Huang, X. F., Hui, L. I., Jian, W. S., \& Gong, J. Y., 2007. A survey of research on accuracy analysis and improvement of airborne laser scanning data. Remote Sensing Information,(3),pp.91-95.

Ju T., 2004.Robust repair of polygonal models. ACM Trans. Graph., 23(3),pp.888-895.

Koller D., Levoy M., 2006.Computer-aided Reconstruction and New Matches in the Forma Urbis Romae. Bullettino Della
Commissione Archeologica Comunale di Roma, Supplementi,15,pp.103-125.

Lerones P M, Llamas J, Gómez-García-Bermejo J, et al., 2014.Using 3D digital models for the virtual restoration of polychrome in interesting cultural sites. Journal of Cultural Heritage, 15(2),pp.196-198.

LI Deren., 2017. From Geomatics to Geospatial Intelligent Service Science. Acta Geodaetica et Cartographica Sinica, 46(10),pp. 1207-1212.

Liu, J. G., 2017. Multi-view 3d reconstruction of shoushengsi tower in wanrong county,shanxi province. Sciences of Conservation \& Archaeology, 29(5), pp.48-51.

Lucia A., Elisabetta S., Marco C., et al., 2013. Innovative uses of $3 \mathrm{D}$ digital technologies to assist the restoration of a fragmented terracotta statue. Journal of Cultural Heritage, 14(4), pp. 332-345.

Noorudin F., Turk G., 2003. Simplification and repair of polygonal models using volumetric techniques. IEEE Transactions on Visualization and Computer Graphics, 9, pp.191-205.

Pan, Y. H., \& Dong-Ming, L. U., 2003. Digital protection and restoration of dunhuang mural. Acta Simulata Systematica Sinica,15(3), pp.310-314.

Shan Jixiang., 2017. Evolution of World's Civilization Patterns and Cultural Heritage Conservation Strategy. Bulletin of Chinese Academy of Sciences, 32(7), pp. 681-689.

Yang, S., \& Wu, Y., 2012. Application of 3-d laser scanning technology in the conservation of cave no.10 at anyue yuanjuedong grottoes. China Cultural Heritage Scientific Research, (2), pp. 74-77.

Yu Y., Zhou K., Xu D., et al., 2004. Mesh editing with poissonbased gradient field manipulation. ACM Transactions on Graphics (TOG). Proceedings of ACM SIGGRAPH, 23(3), pp. 644-651.

Zhou, P. B., Jun-Nan, L., \& Shui, W. Y., 2014. Virtual restoration of broken artifacts based on fracture surface. Journal of System Simulation, 26(9), pp. 2176-2179.

Zhang, R., Luo, Y. L., Zhou M.Q., 2007. The key technology in digital cultural relics. Journal of Beijing Normal University, 43(2), pp. 150-153.

Zhang, Y. H., Geng, G. H., Wei, X. R., Zhang, J., \& Zhou, M. Q., 2017. Reassembly of fractured fragments based on skeleton graphs matching. Zidonghua Xuebao/acta Automatica Sinica, 43(4), pp. 622-633. 